\title{
Editorial: Electrocatalysis on Shape-Controlled Nanoparticles
}

\author{
Paramaconi Rodriguez ${ }^{1 *}$ and José Solla-Gullón ${ }^{2 *}$ \\ ${ }^{1}$ School of Chemistry, University of Birmingham, Birmingham, United Kingdom, ${ }^{2}$ Institute of Electrochemistry, University of \\ Alicante, Alicante, Spain
}

Keywords: electrochemistry, electrocatalysis, shape control, nanoparticles, surface structure sensitivity

\section{Editorial on the Research Topic}

\section{Electrocatalysis on Shape-Controlled Nanoparticles}

The incorporation of shape-controlled metal nanoparticles in electrocatalysis is improving the activity, selectivity, and stability of many different electrocatalytic reactions of interest (Hong et al., 2016; Strasser et al., 2018; García-Cruz et al., 2019; Rizo and Roldan Cuenya, 2019). It is well-established, by simple crystallographic analogies, that the shape of a nanoparticle strongly determines the coordination of the atoms at its surface; that is, its surface structure (Zhou et al., 2011). Consequently, the application of such shaped nanoparticles is also deeply contributing to a better understanding of the effect of the surface structure at nanoscale for many electrocatalytic processes. However, despite significant contributions, much more work is still required to fully visualize the capabilities of these nanomaterials (Pan et al., 2019).

Among the factors that must be taken into consideration, the surface cleanliness is one of the most critical. Several synthetic approaches can produce shaped nanoparticles, but the use of surface capping agents under colloidal routes is one of the most effective ways to control the growth of nanoparticles so as to induce the formation of specific particle shapes (Xia et al., 2015; Yang et al., 2019). However, once nanoparticles are formed, the presence of these surface-regulating agents at the surface of the nanoparticles results in an obstacle; and it is mandatory to apply specific decontamination protocols to completely remove (without altering the initial surface structure of the nanoparticles) their presence from the surface of the nanoparticles (Monzó et al., 2012; Montiel et al., 2017; García-Cruz et al., 2019). This is a critical step that has been generally underestimated in literature dealing with the electrocatalytic properties of shaped nanoparticles. Devivaraprasad et al. reported a comprehensive review dealing with some of the most important aspects of the use of shaped Pt and Pd nanoparticles including, (i) methods of synthesis of these nanoparticles, (ii) decontamination procedures to effectively remove the adsorbed capping agents and/or surfactants without disturbing the surface structure, (iii) electrochemical reactivity of these shaped nanoparticles toward the oxygen reduction reaction (ORR), and (iv) stability of the nanoparticles under electrochemical conditions. This review presents an ideal overview of all aspects which must be taken into consideration to properly employ these nanoparticles for any electrocatalytic study.

Getting detailed and statistically representative information about the surface structure of the shaped nanoparticles is also of fundamental importance because the key point controlling the electrocatalytic activity is the surface structure of the nanoparticles (obviously, the atomic composition is also extremely relevant), not the particle shape (Vidal-Iglesias et al., 2011). In this regard, the use of electrochemical tools has allowed a detailed characterization of the surface structure of different shaped Pt and Au nanoparticles (Rodríguez et al., 2005; SollaGullón et al., 2008; Monzo et al., 2015; Jeyabharathi et al., 2018). Garnier et al. discussed the use of the $\mathrm{Cu}$ UPD to characterize the surface structure of different shape controlled Pd 
nanoparticles. The results obtained indicate that $\mathrm{Cu}$ UPD allows, based on Pd single crystal features, a qualitative and quantitative analysis of the surface structure of different shapeand-size-controlled Pd nanoparticles to be performed. This detailed characterization is of fundamental interest to properly establishing and understanding the correlations between electrocatalytic activity and surface structure at nanoscale.

The modification of the surface of the shaped nanoparticles with some adatoms is also an interesting approach to enhance the activity, stability, and selectivity of different electrocatalytic reactions of interest (de Souza et al., 2018; Dionigi et al., 2019). Rafaïdeen et al. studied the electrooxidation of glucose in alkaline medium on bare and Au-modified shapecontrolled Pd nanoparticles. On non-modified nanoparticles, those with a higher density of $\{100\}$ domains displayed higher activity. For the Au-modified samples, and independent of the particle shape, the resulting activity is much higher than that obtained with bare samples; although, the activity is clearly dependant on the $\mathrm{Au}$ coverage. However, the Aumodified Pd nanocubes still exhibited the highest activity toward glucose electrooxidation. Under the same concept, Hong et al. reported the preparation of $\mathrm{Pt}-\mathrm{Cu}$ octahedral nanoparticles decorated with $\mathrm{Ni}(\mathrm{OH})_{2}$. The authors also evaluated the catalytic activity of the nanocatalyst toward the electrochemical oxidation of ethanol and carbon monoxide in acidic solution. It is shown that the $\mathrm{Ni}(\mathrm{OH})_{2}$-decorated samples displayed enhanced $\mathrm{CO}$ tolerance relative to the bare nanoparticles, due to the presence of $\mathrm{Ni}(\mathrm{OH})_{2}$ at their surface. Even more, both $\mathrm{Ni}(\mathrm{OH})_{2}$-decorated and bare Pt$\mathrm{Cu}$ octahedral samples show enhanced activity and durability during ethanol electrooxidation, in comparison with commercial $\mathrm{Pt} / \mathrm{C}$ electrocatalysts.

Developing non-platinum-group-metal (PGM)-based electrocatalysts is also of great interest; in particular, as catalysts for the ORR in Polymer Electrolyte Membrane Fuel Cells

\section{REFERENCES}

de Souza, M. B. C., Fernández, P. S., and Solla-Gullón, J. (2018). Adatom decorated shape-controlled metal nanoparticles: advanced electrocatalysts for energy conversion. Curr. Opin. Electrochem. 9, 121-128. doi: 10.1016/j.coelec.2018.03.007

Dionigi, F., Weber, C. C., Primbs, M., Gocyla, M., Bonastre, A. M., Spöri, C., et al. (2019). Controlling near-surface Ni composition in octahedral PtNi(Mo) nanoparticles by Mo doping for a highly active oxygen reduction reaction catalyst. Nano Lett. 19, 6876-6885. doi: 10.1021/acs.nanolett.9b02116

García-Cruz, L., Montiel, V., and Solla-Gullón, J. (2019). Shape-controlled metal nanoparticles for electrocatalytic applications. Phys. Sci. Rev. 4:20170124. doi: 10.1515/psr-2017-0124

Greeley, J., Jaramillo, T. F., Bonde, J., Chorkendorff, I., and Nørskov, J. K. (2006). Computational high-throughput screening of electrocatalytic materials for hydrogen evolution. Nat. Mater. 5, 909-913. doi: 10.1038/nmat1752

Hong, J. W., Kim, Y., Kwon, Y., and Han, S. W. (2016). Noble-Metal nanocrystals with controlled facets for electrocatalysis. Chem. Asian J. 11, 2224-2239. doi: 10.1002/asia.201600462

Jeyabharathi, C., Zander, M., and Scholz, F. (2018). Underpotential deposition of lead on quasi-spherical and faceted gold nanoparticles. J. Electroanal. Chem. 819, 159-162. doi: 10.1016/j.jelechem.2017.10.011
(Rabis et al., 2012). Khan et al. reported the use of a novel, two-dimensional, mesoporous $\mathrm{CrN}$-reduced graphene oxide (rGO) nanocomposite with $\mathrm{MnO}$ as an electrocatalyst toward the ORR in alkaline solution. This non-PGM-based electrocatalyst displays enhanced ORR properties relative to a benchmark $20 \%$ wt $\mathrm{Pt} / \mathrm{C}$ electroacatalyst. In addition, the nanocomposite also shows an interesting methanol tolerance and improved stability in alkaline media. Preliminary fuel cell testing [alkaline anion exchange membrane fuel cell (AAEMFC)] provided a maximum power density of about $309 \mathrm{~mW} \mathrm{~cm}^{-2}$, surpassing the maximum power of $250 \mathrm{~mW} \mathrm{~cm}^{-2}$, obtained with the benchmark $20 \% \mathrm{wt}$ $\mathrm{Pt} / \mathrm{C}$ electroacatalyst.

Theoretical calculations are strongly impacting and guiding the development of new electrocatalysts (Greeley et al., 2006; Nørskov et al., 2009). Lozano and Rankin reported relevant DFT calculations, studying the effect of particle size and the incorporation of some dopants in different graphenenanoparticle systems. The adsorption energy of mono-atomic oxygen $\mathrm{O}^{*}$ was used as descriptor of the ORR activity. Several sub-nanometer (4, 7, and 19-atoms) binary alloy/intermetallic transition metal nanoparticle (Ni-Cu, Au-Pd, Rh-Ir, Cu-Ni, and Pt-Pd) catalysts on different doped graphene were studied. The results obtained with doped-graphene- $\mathrm{Ni}_{\mathrm{x}} \mathrm{Cu}_{\mathrm{y}}$ systems were found to provide the necessary shifts in $\mathrm{O}^{*}$ binding energies to reach the predicted activity maximum in the volcano plot for the ORR.

As Guest Editors of this Research Topic, we would like to thank all the authors and reviewers for their valuable contributions to this special issue. We expect that the readers find this topic issue of interest.

\section{AUTHOR CONTRIBUTIONS}

All authors listed have made a substantial, direct and intellectual contribution to the work, and approved it for publication.

Montiel, M. A., Vidal-Iglesias, F. J., Montiel, V., and Solla-Gullón, J. (2017). Electrocatalysis on shape-controlled metal nanoparticles: progress in surface cleaning methodologies. Curr. Opin. Electrochem. 1, 34-39. doi: 10.1016/j.coelec.2016.12.007

Monzó, J., Koper, M. T. M., and Rodriguez, P. (2012). Removing polyvinylpyrrolidone from catalytic Pt nanoparticles without modification of superficial order. ChemPhysChem 13, 709-715. doi: 10.1002/cphc.201 100894

Monzo, J., Malewski, Y., Vidal-Iglesias, F. J., Solla-Gullon, J., and Rodriguez, P. (2015). Electrochemical oxidation of small organic molecules on $\mathrm{Au}$ nanoparticles with preferential surface orientation. Chemelectrochem 2, 958-962. doi: 10.1002/celc.201500084

Nørskov, J. K., Bligaard, T., Rossmeisl, J., and Christensen, C. H. (2009). Towards the computational design of solid catalysts. Nat. Chem. 1, 37-46. doi: 10.1038/nchem.121

Pan, L., Ott, S., Dionigi, F., and Strasser, P. (2019). Current challenges related to the deployment of shape-controlled Pt alloy oxygen reduction reaction nanocatalysts into low Pt-loaded cathode layers of proton exchange membrane fuel cells. Curr. Opin. Electrochem. 18, 61-71. doi: 10.1016/j.coelec.2019.10.011

Rabis, A., Rodriguez, P., and Schmidt, T. J. (2012). Electrocatalysis for polymer electrolyte fuel cells: recent achievements and future challenges. ACS Catal. 2, 864-890. doi: $10.1021 / \operatorname{cs} 3000864$ 
Rizo, R., and Roldan Cuenya, B. (2019). Shape-controlled nanoparticles as anodic catalysts in low-temperature fuel cells. ACS Energy Lett. 4, 1484-1495. doi: 10.1021 /acsenergylett.9b00565

Rodríguez, P., Herrero, E., Solla-Gullón, J., Vidal-Iglesias, F. J., Aldaz, A., and Feliu, J. M. (2005). Specific surface reactions for identification of platinum surface domains - Surface characterization and electrocatalytic tests. Electrochim. Acta 50, 4308-4317. doi: 10.1016/j.electacta.2005.02.087

Solla-Gullón, J., Rodríguez, P., Herrero, E., Aldaz, A., and Feliu, J. M. (2008). Surface characterization of platinum electrodes. Phys. Chem. Chem. Phys. 10, 1359-1373. doi: 10.1039/b709809j

Strasser, P., Gliech, M., Kuehl, S., and Moeller, T. (2018). Electrochemical processes on solid shaped nanoparticles with defined facets. Chem. Soc. Rev. 47, 715-735. doi: $10.1039 / \mathrm{c} 7 \mathrm{cs} 00759 \mathrm{k}$

Vidal-Iglesias, F. J., Solla-Gullón, J., Herrero, E., Montiel, V., Aldaz, A., and Feliu, J. M. (2011). Evaluating the ozone cleaning treatment in shape-controlled Pt nanoparticles: evidences of atomic surface disordering. Electrochem. Commun. 13, 502-505. doi: 10.1016/j.elecom.2011.02.033

Xia, Y., Xia, X., and Peng, H.-C. (2015). Shape-controlled synthesis of colloidal metal nanocrystals: thermodynamic versus kinetic products. J. Am. Chem. Soc. 137, 7947-7966. doi: 10.1021/jacs.5b04641
Yang, T.-H., Shi, Y., Janssen, A., and Xia, Y. (2019). Surface capping agents and their roles in shape-controlled synthesis of colloidal metal nanocrystals. Angew. Chem. Int. Ed. doi: 10.1002/anie.201 911135

Zhou, Z. Y., Tian, N., Li, J. T., Broadwell, I., and Sun, S. G. (2011). Nanomaterials of high surface energy with exceptional properties in catalysis and energy storage. Chem. Soc. Rev. 40, 4167-4185. doi: 10.1039/C0CS 00176G

Conflict of Interest: The authors declare that the research was conducted in the absence of any commercial or financial relationships that could be construed as a potential conflict of interest.

Copyright (c) 2019 Rodriguez and Solla-Gullón. This is an open-access article distributed under the terms of the Creative Commons Attribution License (CC BY). The use, distribution or reproduction in other forums is permitted, provided the original author(s) and the copyright owner(s) are credited and that the original publication in this journal is cited, in accordance with accepted academic practice. No use, distribution or reproduction is permitted which does not comply with these terms. 